\title{
The long war against flu
}

\author{
That the H5N1 strain of bird flu has not yet caused a pandemic is no cause for complacency. Preparations \\ for the inevitable must be redoubled to mitigate the potential devastation.
}

ive years after the deadly H5N1 avian influenza virus exploded into a global epidemic in birds, it has infected more than 300 people. Happily, it has not yet evolved into a strain that can transmit easily between humans - an event that would trigger a pandemic that could kill tens of millions. But as long as H5N1 continues to be present in animals, that risk persists. And with so many other flu strains out in the world, all constantly evolving, a flu pandemic is inevitable.

This grim reality has spurred basic research into topics such as the 1918 flu virus, cell-receptor biology and evolutionary dynamics, which are collectively yielding insights into the molecular basis of virulence and how viruses adapt to humans. Researchers have also begun to unravel the often fatal clinical events caused by the virus, such as the massive immune response that is a 'cytokine storm', and cell-culture technology is promising to make vaccines available more quickly. Plans by the Bill \& Melinda Gates Foundation, the Wellcome Trust and the Pasteur Institute to roadmap this research should help focus priorities for funding, just as similar work has done for neglected diseases.

But improved control measures, especially for H5N1 itself, and public-health infrastructure are our frontline defences against a pandemic. Unfortunately, the overall control picture is bleak. Thailand, Vietnam and China have notched up successes in curbing outbreaks in birds, which is key to minimizing the chance that the virus can pass to humans. But South Korea had its worst outbreak ever in April, and the disease has become endemic in Indonesia, Bangladesh, Vietnam and Egypt. Eradication now seems impossible, and the task of containing the virus has become chronic and costly.

Many countries have made patchy progress in planning how to mitigate a pandemic once it does break out. True, any such plan can only buy time, by using antiviral drugs and restricting movement, until a vaccine is available for the specific strain that has broken out. The Commentary on page 162 endorses what might be an intriguing adjunct: 'pre-pandemic' vaccines, which would be matched not to the exact pandemic strain, but to earlier variants. Even if these vaccines were only partly effective, advocates argue, they might confer sufficient protection to prevent death or severe disease. Although this idea is untested, it merits consideration - especially as strain-specific vaccines would be available only several months into the pandemic, and even then would be in very short supply. The World Health
"Even if H5N1 never evolves into a pandemic strain, it serves as a useful wake-up call." Organization is planning to stockpile more than 100 million doses of pre-pandemic vaccines, and some nations, including Japan, are considering the same.

But delivering sufficient perfectly matched pandemic vaccine fast enough to make a difference is the critical issue. One promising approach - equipping vaccines with adjuvants that boost their effect, reducing the amount of antigen needed in each dose - is belatedly getting the attention it deserves. Indeed, research is generating vaccine formulations that need so little antigen that timely doses could, in principle, be provided for everyone on Earth using existing plant capacity.

Rapid delivery will require an unprecedented level of international coordination. Plans should be in place so that when a pandemic strikes clinical trials of the strain-specific vaccine begin - as do the manufacture and distribution of the billions of syringes needed to deliver it. There should also be international mechanisms to ensure that developing countries have access to pandemic and pre-pandemic vaccines at low cost.

Surveillance, control of disease in animals, pandemic planning and vaccines - each requires intense, organized and sustained commitment. Even if $\mathrm{H} 5 \mathrm{~N} 1$ never evolves into a pandemic strain, it serves as a useful wake-up call, revealing just how much more must be done to be better prepared for the inevitable.

\section{An unnecessary battle}

\section{Neuroscientists and geneticists don't need to be at loggerheads over the biology of mental disorders.}

M ental disorders take a staggering health and economic toll. The World Health Organization has estimated that unipolar depressive disorder alone is one of the leading causes of disability worldwide. Schizophrenia, bipolar disorder, autism and the many other psychiatric disorders only add to the misery. Yet progress in understanding the underlying causes of these conditions seems to be moving at a crawl. Genes are surely involved, but decades of futile hunting have made it painfully clear that the contribution of any single gene to disease is probably minuscule.

How to find these tenuous connections is a contentious scientific debate, with geneticists and neuroscientists at an apparent impasse (see page 154). These two communities must start working together more constructively if they are to crack this challenging problem and ensure that the millions of dollars now flooding into this field are not misspent.

Many geneticists believe that scanning the entire genomes of a massive number of patients will uncover weak gene candidates by sheer statistical power. Yet some neuroscientists dismiss these studies, questioning the utility of indiscriminately seeking a swath of genes that are weakly associated with a condition, and that are unlikely to have relevance to its biology. Another concern is that diagnoses of psychiatric 
disorders are often based on fuzzy, subjective and inconsistent criteria. The neuroscientists feel that such untargeted searches will be futile if the population labelled as having one condition — such as schizophrenia - in fact includes many different clinical disorders.

The antipathy is mutual. Some neuroscientists take what is to a geneticist an unacceptably small number of subjects - sometimes not even patients - and focus on establishing links between variants of a candidate gene and brain anatomy or function. Geneticists are often not persuaded by the statistics and question the focus on a few candidate genes whose link to disease has never been well established. Neither side seems to agree that the outcomes of the other's studies represent constructive leads. Nor is either side willing to compromise its standards. As a result, it is difficult to publish in the intersection between these two fields.

This conflict is unnecessary. Both communities will gain by learning from the other. Teams undertaking expensive genome-wide association studies should consult fully with clinical and research neuroscientists. If a single diagnostic label such as 'schizophrenia' is inadequate, then more detailed clinical information on patients must be collected when they are recruited. The studies might also benefit from more quantifiable measures of behaviour and neurobiology - such as laboratory assays of anxiety or the functioning of fear circuits in the brain.

And neuroscientists making early forays into genetic associations may find the hard lessons learned by geneticists over the years useful. Geneticists know about the statistical methods and criteria required to draw genetic conclusions that are persuasive and likely to stand the test of time.

The two sides should not lose sight of the fact that they share the same goal: to help patients. In the end, it is likely that both strategies and others will prove valuable in identifying risk factors and developing ways to counteract them. If genome-wide scans are appropriately designed and powered, they should yield the first clues. The neuroscientists' approach may then prove fruitful by showing what biological function is being performed by the genes identified, how that function leads to disease and how it can be altered.

When two fields such as these come together, lowering standards is not an option. Extracting psychiatric genes will require highly rigorous strategies, and on that geneticists and neuroscientists can agree.

\section{A social contract}

\section{Efforts to inform US military policy with insights from the social sciences could be a win-win approach.}

G iven the current US administration's notorious lack of respect for science, the efforts of defence secretary Robert Gates merit a mention. Gates took charge of the Pentagon in December 2006 and ever since has been trying to inform the military's thinking with researchers' insights into other cultures and societies. In October, for example, the Department of Defense launched the Human Terrain System (HTS) programme, in which teams of social scientists advise US troops operating in Iraq and Afghanistan. And on 30 June, the defence department signed a memorandum of understanding that will direct some of its money into social and behavioural science research through the National Science Foundation (NSF).

Gates's outreach may owe something to his tenure as president of Texas A\&M University in College Station for the four years before his current appointment. But it certainly reflects the hard lessons of Iraq and Afghanistan - where troops with insufficient understanding of the cultural or political landscape have too often exacerbated the insurgency they were trying to control.

With an HTS team, an anthropologist, say, might be present to advise a commander on the subtleties of negotiating with village elders in rural Afghanistan. According to Gates, one commander in Afghanistan says that using an HTS team has cut the number of armed attacks he has had to make by $60 \%$.

The recent NSF deal aims to shape the Pentagon's long-term strategic thinking by funding academic research in areas such as religious fundamentalism, terrorism and cultural change. Gates also hopes that such research could foster entirely new intellectual tools, in much the same way that work during the cold war fostered game theory.
All proposals will be selected for funding through the NSF's standard peer-review process. The research will be unclassified and no restrictions will be placed on researchers' freedom to publish their results - or for that matter, to criticize the defence department.

The two initiatives have received a decidedly mixed response. Some social scientists have enthusiastically embraced the goals of the HTS programme. But the American Anthropological Association (AAA) has formally condemned it, saying that participants would find it difficult or impossible to follow the association's ethical guidelines in a combat zone, and that it could make it more difficult for anthropologists to build trust elsewhere in the world.

The NSF agreement has been widely acclaimed by university administrators, who welcome the extra research money. But it has aroused the suspicion among some researchers that it will distort social science towards military priorities. Of particular concern is the fact that the defence department will have some say in the choice of the NSF's peer reviewers.

So far, Gates and his deputies have tried to be sensitive to these concerns. But continued vigilance is paramount. War is notoriously fraught with ambiguity and moral compromise, and it may well be a temptation for commanders to use the information gathered by HTS researchers to, say, target suspect populations. Such temptations should be resisted wherever possible: in the long run, honest dealings and the do-no-harm principle required by the AAA are in the military's own best interest. So too is avoiding any temptation to load the NSF peer-review panels.

Social scientists, meanwhile, should embrace the opportunities that the AAA pointed out last November in a report on engagement with the military. These include studying military and intelligence organizations from the inside and educating the military about other cultures and societies. Outrage at the current administration should not derail efforts that have potential to be a win-win for all concerned - including, most especially, the people of Iraq, Afghanistan and regions of future conflict. 